\title{
Quelle valeur attribuer à l'analyse morphologique des spermatozoïdes en microscopie optique?
}

\author{
Marie-Roberte GUICHAOUA1,2, Jeanne PERRIN1,2, \\ Cendrine GEOFFROY-SIRAUDIN1,2, Mireille PAPADACCI1 \\ 1 Laboratoire de Biologie de la Reproduction, Hôpital de la Conception, Marseille \\ 2 EA 1784 Biogénotoxicologie et mutagenèse environnementale, Marseille
}

\section{RESUME}

La morphologie des spermatozoïdes reste le paramètre qui suscite le plus de contradictions dans la littérature lorsque l'on veut tenter d'en apprécier la valeur prédictive. II est cependant clair qu'actuellement on ne peut pas écarter la morphologie des spermatozoïdes comme critère d'appréciation de la qualité de ces cellules, cet examen reste d'une grande importance dans le bilan d'une infertilité masculine ou du couple. Par ailleurs, les nouvelles technologies, qui permettent de voir et donc de sélectionner sur leur morphologie les spermatozoïdes avec des grossissements supérieurs à ceux utilisés jusqu'ici, améliorent considérablement les résultats des fécondations in vitro.

Plusieurs systèmes de classification détaillée des anomalies des spermatozoïdes ont été proposés depuis une cinquantaine d'années. Ces classifications deviennent de plus en plus strictes à chacune de leurs révisions. Trois parmi ces classifications servent le plus fréquemment de référence : la classification de Kruger/Tygerberg et la classification de David, redéfinie avec un soin particulier dans le souci de la démarche d'assurance en qualité en biologie de la reproduction par Auger et Eustache. Les résultats de ces analyses sont très hétérogènes en ce qui concerne le pourcentage global d'anomalies morphologiques et les fréquences respectives des différentes anomalies. II est donc important de souligner qu'il est indispensable d'apporter un soin particulier à la réalisation technique de cet examen, à l'établissement de critères stricts d'appréciation de chaque anomalie et à l'harmonisation de ces critères entre les divers observateurs d'un même laboratoire et entre les laboratoires.

L'impact de la tératozoospermie isolée sur les résultats de la FIV et de l'ICSI fait l'objet de nombreuses études dont les résultats sont là encore parfois contradictoires. Cependant, la majorité des études montre que le pourcentage de spermatozoïdes morphologiquement normaux est positivement corrélé avec les résultats de l'ICSI, et de nombreux auteurs admettent qu'un pourcentage de spermatozoïdes morphologiquement normaux inférieur à $5 \%$ est prédictif d'un taux faible de fécondation et de grossesse en FIV et en ICSI.
Au cours de ces dix dernières années, on a montré que les taux d'aneuploïdies dans le sperme des populations d'hommes infertiles présentant une oligospermie sévère ou modérée étaient plus élevés que ceux des hommes fertiles avec spermogramme normal, et que les taux de disomies des spermatozoïdes étaient environ 20 fois plus élevés dans les ICSI que dans les FIV. Cependant, les résultats de ces différentes études ne permettent pas d'établir un lien évident entre la tératozoospermie polymorphe et la fréquence des disomies et des aneuploïdies dans le sperme.

Globalement, on peut conclure que l'analyse morphologique des spermatozoïdes en microscopie optique, au grossissement habituellement utilisé pour la réalisation des spermocytogrammes $(\times 2000)$, n'est donc pas un bon indicateur des anomalies chromosomiques dans le sperme humain, excepté dans quelques cas rares d'anomalies monomorphes. II n'en est pas de même des liens entre la morphologie des spermatozoïdes et l'apoptose. En effet, un nombre croissant de travaux établissent une corrélation positive entre l'infertilité masculine et la présence de marqueurs apoptotiques sur les spermatozoïdes, et le paramètre morphologie semble particulièrement lié à l'apoptose. Enfin, il est important de souligner l'absolue nécessité d'une standardisation des procédures employées et de tous les résultats de l'analyse morphologique du sperme.

Mots clés : morphologie de spermatozoïdes, infertilité, anomalies chromosomiques, apoptose, FIV, ICSI

\footnotetext{
Correspondance :

Pr Marie-Roberte GUICHAOUA - Laboratoire de Biologie de la Reproduction, Hôpital de la Conception, 147 Boulevard Baille, 13585, Marseille Cedex 5 -

Email mguichaoua@hp-hm.fr
} 


\section{INTRODUCTION}

La morphologie des spermatozoïdes est le paramètre qui suscite le plus de contradictions dans la littérature lorsque l'on veut tenter d'en apprécier sa valeur prédictive. En effet, si l'analyse de la morphologie des spermatozoïdes en microscopie optique ne peut pas rendre compte avec certitude du pouvoir fécondant des spermatozoïdes [31, 37, 46, 48], elle constitue une étape obligatoire de l'établissement du diagnostic d'infécondité masculine. L'analyse du phénotype des spermatozoïdes peut révéler une atteinte de la spermatogenèse et/ou de la fonction épididymaire.

Dès 1976, David et al. [16], en utilisant des critères rigoureux d'analyse de la morphologie des spermatozoïdes, ont mentionné qu'une tératospermie importante pouvait par elle-même compromettre la valeur fécondante d'un sperme. Puis, Kruger et al. $[26,27]$ ont montré que les taux de fécondation sont significativement diminués lorsque le pourcentage de spermatozoïdes morphologiquement normaux est inférieur à $14 \%$, et que les chances de fécondation sont compromises au dessous de $5 \%$ de formes typiques. Des travaux plus récents soulignent aussi la valeur pronostique in vivo et in vitro du spermocytogramme, certains auteurs n'hésitant pas à affirmer que la morphologie des spermatozoïdes est le meilleur indicateur de la fertilité masculine $[7,15,23,33,35,39,49,50]$.

D'autres études contredisent ces résultats. Dans un travail dédié à l'impact des caractéristiques du sperme dans l'évaluation de la fertilité versus l'infertilité masculine, Nallella et al. [38] suggèrent que la morphologie des spermatozoïdes est un moins bon critère de discrimination que la concentration ou la mobilité. L'analyse comparative des résultats des FIV conventionnelles et des ICSI avec les pourcentages des spermatozoïdes présentant des formes typiques [25] aboutit à la même conclusion, à savoir que la tératospermie isolée n'a pas d'impact majeur sur les résultats des fécondations in vitro (taux de fécondation et de grossesse, taux de naissances vivantes).

Il est cependant clair qu'actuellement on ne peut pas écarter la morphologie des spermatozoïdes comme critère d'appréciation de la qualité de ces cellules. En effet, les nouvelles technologies, qui permettent de voir et donc de sélectionner sur leur morphologie les spermatozoïdes avec des grossissements supérieurs à ceux utilisés jusqu'ici, améliorent considérablement les résultats des fécondations in vitro [5].

Par ailleurs, il est maintenant évident que l'on ne peut plus parler de tératospermie au singulier, l'analyse de la littérature montre qu'il existe en réalité deux grands groupes de tératospermies : les tératospermies polymorphes, de loin les plus fréquentes, dans lesquelles les anomalies sont réparties sur les différentes régions du spermatozoïde, tête, collet, pièce intermédiaire et flagelle, et les tératospermies monomorphes où une ou plusieurs anomalies particulières est/sont présente(s) sur la totalité ou un pourcentage élevé de spermatozoïdes. Ces tératospermies monomorphes constituent des syndromes maintenant bien définis, tels que la globozoospermie, le syndrome des spermatozoïdes macrocéphales, le syndrome des spermatozoïdes décapités ou les anomalies du flagelle, leur présence oriente la conduite à tenir pour le choix d'une technique d'assistance médicale à la procréation (AMP) et pour le suivi de la grossesse, ou constitue une contre-indication à l'utilisation de ce sperme pour toute tentative d'AMP.
Cependant, des formes atypiques de ces syndromes ont été décrites, en particulier, des formes dans lesquelles coexistent des spermatozoïdes normaux et des spermatozoïdes présentant l'anomalie d'intérêt (formes partielles) plus difficiles à diagnostiquer, et que nous décrirons en détail dans l'article consacré aux anomalies monomorphes des spermatozoïdes (Metzler-Guillemain et al., 46-49).

Bien qu'il existe une part de subjectivité dans l'analyse morphologique des spermatozoïdes, il est donc incontestable que cet examen reste d'une grande importance dans l'appréciation de la qualité du sperme et le bilan d'une infertilité masculine ou du couple. II est aussi évident qu'il faut distinguer entre les valeurs moyennes obtenues dans une population normale et les paramètres minimum nécessaires pour obtenir une conception. Enfin, il est important de souligner qu'il est indispensable d'apporter un soin particulier à la réalisation technique de cet examen, à l'établissement de critères stricts d'appréciation de chaque anomalie et à l'harmonisation de ces critères entre les divers observateurs d'un même laboratoire et entre les laboratoires.

\section{MORPHOLOGIE DES SPERMATOZOIIDES DANS LES POPULATIONS D'HOMMES FERTILES}

Plusieurs systèmes de classification détaillée des anomalies des spermatozoïdes ont été proposés depuis une cinquantaine d'années : Williams [52], Freund [21], Eliasson [17], David [16], Fredericsson [20], critères stricts de Tygerberg [26, 27, 28], Organisation Mondiale de la Santé (OMS) [53-56].

Ces classifications deviennent de plus en plus strictes à chacune de leurs révisions. Trois parmi ces classifications servent le plus fréquemment de référence : la classification de Kruger/Tygerberg [28], la classification de l'OMS [55] et celle que nous utilisons préférentiellement en France, la classification de David [16], redéfinie avec un soin particulier et dans le souci de la démarche d'assurance en qualité en biologie de la reproduction par Auger et Eustache [2]. Nous renvoyons donc le lecteur à cet article pour la description précise des anomalies des spermatozoïdes observées dans un spermocytogramme, préférant nous arrêter à leurs fréquences respectives dans un éjaculât normal et aux conséquences que peut avoir pour le pouvoir fécondant du sperme ou pour la qualité embryonnaire l'augmentation de fréquence de ces anomalies.

Malgré l'intérêt que nous semble présenter l'analyse du spermocytogramme, il existe peu de publications détaillant les fréquences de chaque anomalie des spermatozoïdes dans les populations d'hommes fertiles, la plupart des études prenant en considération le pourcentage global d'anomalies sans tenir compte de la fréquence de chacune de ces anomalies.

Kubo-Irie et al. [29] et Haidl et Schill [22] évaluent le pourcentage de spermatozoïdes normaux chez les hommes fertiles à respectivement $35,5 \%$ et $30 \%$. En France, on parle de tératospermie lorsque le pourcentage de spermatozoïdes morphologiquement normaux est égal ou inférieur à $30 \%$, à partir des critères définis par la classification de David [16]. Dans le manuel de l'OMS [52], le seuil de spermatozoïdes normaux, évalué sur des critères décrits dans ce manuel, est de $15 \%$, il est de $14 \%$ pour Krugger [27]. Cinq études détaillent les pourcentages de chacune des anomalies décrites au niveau des spermatozoïdes chez les hommes fertiles $[10,16,29,42$ et données de notre laboratoire] (Tableau 1). Nous rapportons ici 
Tableau 1 : Pourcentages respectifs des différentes anomalies morphologiques des spermatozoïdes dans le sperme normal [16, 42, 10, 29, et Guichaoua et al. (janvier 2005 à octobre 2007)].

\begin{tabular}{|c|c|c|c|c|c|}
\hline & $\begin{array}{l}\text { David } \\
1975 \\
{[16]}\end{array}$ & $\begin{array}{c}\text { Schwartz } \\
1984 \\
{[42]}\end{array}$ & $\begin{array}{l}\text { Bujan } \\
1988 \\
{[10]}\end{array}$ & $\begin{array}{c}\text { Kubo-Irie } \\
2004 \\
{[29]}\end{array}$ & $\begin{array}{c}\text { Guichaoua } \\
2007 \\
{\left[{ }^{*}\right]}\end{array}$ \\
\hline Formes typiques & $65.76 \pm 10.33$ & 60.3 & 72.7 & 35.5 & $47.8 \pm 11.28$ \\
\hline Tête allongée & $\underline{6.88 \pm 8.13}$ & 5.9 & 1.9 & $4.0 \pm 3.9$ & $1.36 \pm 2.05$ \\
\hline Tête amincie & $1.54 \pm 1.70$ & 4.6 & 2.6 & $2.4 \pm 1.9$ & $2.03 \pm 2.52$ \\
\hline Microcéphale & $5.18 \pm 6.00$ & 9.8 & 2.6 & $18.5 \pm 5.5$ & $2.69 \pm 3.08$ \\
\hline Macrocéphale & $1.72 \pm 1.75$ & 1.6 & 0.9 & $7.0 \pm 4.3$ & $0.94 \pm 1.32$ \\
\hline Tête multiple & $1.20 \pm 1.80$ & 0.4 & 0.7 & $0.3 \pm 0.4$ & $1.78 \pm 1.93$ \\
\hline $\begin{array}{l}\text { Tête irrégulière } \\
\text { Base anormale } \\
\text { Acrosome malformé } \\
\text { Acrosome absent }\end{array}$ & $\begin{array}{c}4.84 \pm 3.18 \\
l \\
l \\
I\end{array}$ & $\begin{array}{c}\frac{15.2}{1} \\
1 \\
1\end{array}$ & $\begin{array}{l}2 \\
! \\
!\end{array}$ & $\begin{array}{c}11.3 \pm 5.5 \\
/ \\
/ \\
/\end{array}$ & $\begin{array}{c}/ \\
\frac{26.38 \pm 11.11}{\frac{28.33 \pm 11.57}{1.88 \pm 2.08}}\end{array}$ \\
\hline Reste cytoplasmique & $5 \pm 4.40$ & 3.2 & 2.5 & $15.1 \pm 9.7$ & $3.48 \pm 3.3$ \\
\hline Angulation & $5.96 \pm 3.78$ & 4.7 & $\underline{5.2}$ & $1.3 \pm 2.0$ & $16.58 \pm 7.08$ \\
\hline$P I$ fine ** & $T$ & 1 & $T$ & $11.5 \pm 10.2$ & $4.0 \pm 5.82$ \\
\hline Flagelle absent & $1.60 \pm 1.59$ & 0.6 & 1.3 & & $2.83 \pm 3.09$ \\
\hline Flagelle court & $0.76 \pm 0.70$ & 1.1 & 0.8 & $6.1 \pm 4.5$ & $2.08 \pm 2.89$ \\
\hline Flagelle enroulé & $6.16 \pm 6.27$ & 5.8 & 7 & $1.3 \pm 2.0$ & $7.96 \pm 5.75$ \\
\hline Flagelle multiple & $0.48 \pm 0.48$ & 0.5 & 0.5 & 1 & $1.24 \pm 1.66$ \\
\hline Lyse & $1.44 \pm 1.52$ & 0.4 & 0 & 1 & $1.66 \pm 2.38$ \\
\hline
\end{tabular}

Les valeurs soulignées en italique représentent les pourcentages les plus élevés dans chaque étude.

* Données non publiées auparavant. Bien que nous utilisions la classification de David modifiée [2], pour plus de clarté du tableau et afin d'homogénéiser la présentation de nos résultats avec ceux des autres auteurs, nous avons regroupé les différents types de têtes allongées de notre étude (simple, base amincie, battant de cloche et effilé) en un seul vocable. Nous avons cependant conservé l'analyse détaillée des têtes irrégulières en base anormale, acrosome malformé, acrosome absent. Les flagelles de calibre irrégulier $(0,24 \pm 0,91)$ n'apparaissent pas dans ce tableau.

${ }^{\star *} P I=$ pièce intermédiaire

les valeurs moyennes normales obtenues dans notre centre à partir de 139 spermogrammes d'hommes fertiles dont les paramètres du spermogramme et le pourcentage de formes typiques sont normaux. Ces hommes ont eu un ou plusieurs bilans de sperme dans le cadre d'un programme d'ICSI pour indication féminine. Trois de ces études utilisent la classification de David non modifiée [10, 16, 42], une quatrième est basée sur la classification de Kruger [35], l'étude de Kubo-Irie et al. [29] repose sur la classification de l'OMS. La plus récente de ces études (Guichaoua et al., données non publiées) utilise la classification de David modifiée (Tableau 1).

Les résultats de ces analyses sont très hétérogènes en ce qui concerne le pourcentage global d'anomalies morphologiques et les fréquences respectives des différentes anomalies (Tableau 1). En effet, le pourcentage de formes typiques dans les cinq études varie de $35,5 \%$ à $72,7 \%$. Dans trois de ces étu- des $[10,16,42]$, les taux de formes typiques sont élevés (respectivement $65,76 \%, 60,3 \%, 72,7 \%$ ) tandis que l'étude de Kubo-Irie et al. [29] ainsi que notre étude montrent des taux plus bas (respectivement $35,5 \%$ et $47,8 \%$ ). L'anomalie la plus fréquemment rencontrée est l'allongement de la tête dans l'étude de David et al. [16], l'irrégularité de la tête dans celle de Schwartz et al. [42] et dans notre étude, l'angulation chez Bujan et al. [10], la microcéphalie dans l'étude de Kubo-Irie et al. [29]. En ce qui concerne l'irrégularité de la tête, son pourcentage varie considérablement entre les études où elles prédominent puisque ce pourcentage est de 15,2\% dans l'étude de Schwartz et al. [42]

A côté de ces variations entre les différentes études, l'analyse des résultats des spermocytogrammes révèle aussi que les pourcentages normaux varient considérablement d'une anomalie à l'autre dans une même ètude. 
Si donc il existe en France un consensus à partir de la classification de David concernant la valeur à partir de laquelle on parle de tératozoospermie, il n'en est pas de même pour le détail des anomalies du spermocytogramme. Il apparaît maintenant nécessaire que les laboratoires de Biologie de la Reproduction établissent leurs normes de référence pour chaque anomalie à partir d'une population d'hommes fertiles dont les spermogrammes sont normaux. Une telle démarche est indispensable pour que soient dépistées les formes partielles de syndromes connus et dans le but d'identifier les anomalies dont la présence en pourcentage modéré constitue un facteur de risque, comme cela est abordé dans d'autres articles du présent numéro consacrés aux anomalies monomorphes [Mitchell et al., pages : 35-45 ; Metzer-Guillemain et al., pages : 46-49 ].

\section{MORPHOLOGIE DES SPERMATOZOÏDES, INFERTILITÉ, FECONDATION IN VITRO}

Nous n'envisagerons dans ce chapitre que les tératospermies polymorphes, les tératospermies monomorphes, qui posent des problèmes très particuliers quant à leurs relations avec l'infertilité et la fécondation in vitro, sont discutées dans d'autres articles du présent numéro [Mitchell et al., pages : 35-45 ; Metzer-Guillemain et al., pages : 46-49]. Toutes les publications qui se sont intéressées au pouvoir fécondant des spermatozoïdes font état d'une corrélation positive entre la morphologie de ces cellules et la fertilité [5-7, 30, 49]. Franken et al. [19] ont montré que la réaction acrosomique induite par la zone pellucide implique préférentiellement les spermatozoïdes dont la morphologie est normale, tandis qu'il existe une corrélation positive significative entre le pourcentage de spermatozoïdes anormaux et la diminution de la capacité de pénétration des spermatozoïdes dans les ovocytes dépellucidés de hamster [9].

\section{Morphologie des spermatozoïdes et infertilité}

II est important d'insister, comme l'ont fait Menkveld [34] et Krugger [27], sur les avantages que présente l'utilisation de critères stricts d'évaluation de la morphologie des spermatozoïdes dans le diagnostic et le pronostic de l'infertilité masculine, la majorité des auteurs utilisant les critères de Kruger/Tygerberg [pour revue, voir 15]. L'analyse morphologique détaillée des spermatozoïdes dans deux populations d'hommes, l'une fertile, l'autre présentant une asthénozoospermie ou une varicocèle, révèle que les mêmes anomalies sont présentes dans la plupart des échantillons des patients fertiles et infertiles, mais que c'est l'incidence globale de ces anomalies qui varie entre les deux populations, les hommes infertiles ayant des taux d'anomalies supérieurs à celles des hommes fertiles [29]. Ainsi, tous les types d'anomalies peuvent être présents dans la plupart des échantillons des patients fertiles et infertiles. II apparaît aussi dans cette étude que les pourcentages de malformations des spermatozoïdes varient d'un individu à l'autre au sein de chacune des deux populations, et que les anomalies du flagelle sont indépendantes de celles de la pièce intermédiaire et de la tête.

\section{Morphologie des spermatozoïdes et fécondation in vitro}

L'impact de la tératozoospermie isolée sur les résultats de la FIV et de l'ICSI fait l'objet de nombreuses études dont les résultats sont là encore parfois contradictoires. Certains auteurs ont tenté d'utiliser la morphologie des spermatozoïdes comme un indicateur de fertilité masculine potentielle dans la FIV, mais aucune étude n'a pu démontrer qu'elle avait une valeur prédictive absolue. Selon Kruger et al., un pourcentage de spermatozoïdes morphologiquement normaux inférieur à $4 \%$ est prédictif d'un taux très faible de fécondation et de grossesse en FIV. Actuellement, un taux discriminatoire de $5 \%$ est admis par de nombreux auteurs [12, 15, 23].

Coetzee et al. (15) ont analysé et fait une synthèse de 10 travaux consacrés aux résultats des FIV chez des couples dont les hommes avaient soit une tératozoospermie sévère $(<5 \%$ de formes typiques) soit un pourcentage de formes typiques $>5 \%$. Les résultats de ces 10 publications montrent que le taux moyen de fécondation dans le groupe de patients avec tératozoospermie sévère ( $<5 \%$ de formes typiques) est inférieur à celui dont le pourcentage de formes typiques est $>5 \%(59,3 \%$ versus $77,6 \%$ ). De même, $24 \%$ des patientes du premier groupe n'ont pas de transfert d'embryons contre $7,4 \%$ dans le deuxième groupe.

A l'inverse, dans un récent travail, Keegan et al. [25] ne montrent aucune différence concernant les taux de fécondation, de grossesse et de naissance vivante au cours de 2 cycles de FIV, entre les couples avec tératospermie isolée et ceux avec sperme normal. Aussi, Aziz et al. [3] ont établi un paramètre d'appréciation de la morphologie des spermatozoïdes appelé SDI (pour Sperm Deformity Index) qui serait un meilleur indicateur de fertilité masculine et de qualité de FIV comparé à l'analyse seule de la morphologie, bien qu'ils prennent en compte les anomalies morphologiques des spermatozoïdes. II n'est cependant pas utilisé en dehors de cette équipe. Le choix de la technique de fécondation, FIV conventionnelle versus ICSI, en fonction du degré de tératozoospermie fait aussi l'objet de discussion étant donné que l'ICSI est une technique plus invasive et consommatrice de temps que la FIV conventionnelle.

La majorité des études montre que le pourcentage de spermatozoïdes morphologiquement normaux est positivement corrélé avec les résultats de l'ICSI [pour revue, voir 14]. Une augmentation des taux de fécondation, d'implantation et de grossesse apparaît dans l'étude de De Vos et al. [51] lorsque les ICSI sont réalisées avec des spermatozoïdes morphologiquement normaux, par rapport aux taux obtenus avec des spermatozoïdes morphologiquement anormaux. Il ne fait aucun doute que la sélection de spermatozoïdes les plus normaux pour l'ICSI améliore les résultats de cette technique. Cependant, Check et al. [12] ont montré que dans les tératozoospermies isolées, bien que le taux de fécondation soit significativement plus élevé dans les ICSI que dans les FIV classiques, les taux de grossesse clinique et de naissance vivante étaient plus élevés dans les FIV classiques que dans les ICSI. Les résultats de cette étude doivent être interprétés avec prudence car il s'agit d'une étude rétrospective faite sur deux échantillons de tailles très différentes. De plus, les auteurs admettent la supériorité de l'ICSI entre 0 et $1 \%$ de formes typiques et soulignent la nécessité de développer le même travail en prospectif afin d'en confirmer les résultats.

Selon Chow et Cheung [14], l'utilisation du seul critère de morphologie des spermatozoïdes dans un but prédictif de fertilité peut conduire à des erreurs diagnostiques et au recours non justifié à l'ICSI. Une stratégie consistant à prôner une réduction du recours à l'ICSI semble avoir actuellement le vent en poupe. Söderlund et Lundin [44] considérent que, les résultats de l'ICSI étant en général inférieurs à ceux de la FIV 
conventionnelle, il ne semble pas nécessaire d'avoir recours à I'ICSI quand la FIV conventionnelle est possible. En effet, 53 cycles dédoublés avec moins de $5 \%$ de formes typiques ne montrent pas de différence significative dans les taux de fécondation obtenus avec la FIV et avec l'ICSI. Leur stratégie permet de réduire de $10 \%$ les indications d'ICSI.

Ainsi, le facteur " forme " des spermatozoïdes en microscopie optique conventionnelle, ne serait pas un facteur fiable dans la sélection des spermatozoïdes fécondants, et pour permettre des taux satisfaisants d'implantation et de grossesse évolutive, en particulier pour l'ICSI. En effet, un certain nombre d'anomalies telles que les vacuoles de la tête ne sont pas détectées en microscopie optique conventionnelle. Nous verrons plus loin qu'il n'en est pas de même lorsque la morphologie des spermatozoïdes est évaluée avec des grossissements supérieurs.

En effet, une remarquable augmentation des taux de grossesse en FIV-IMSI (Intracytoplasmic Morphologically Selected Sperm Injection) est obtenue en injectant les spermatozoïdes ayant les noyaux présentant la plus stricte intégrité morphologique [5]. Un article est consacré dans le présent numéro d'Andrologie [Albert et al., pages : 26-40] à une nouvelle méthode de sélection en temps réel des spermatozoïdes non colorés par l'analyse à fort grossissement des organites des spermatozoïdes mobiles ou MSOME (Motile Sperm Organellar Examination).

\section{ANOMALIES DE LA TÊTE DES SPERMATOZOÏDES ET ANOMALIES DU CONTENU CHROMOSOMIQUE}

La fréquence des anomalies de nombre des chromosomes dans le sperme des hommes fertiles est de 1 à $2 \%$, celle des anomalies de structure varie de 7 à $14 \%$. Au cours de ces dix dernières années, la plupart des études ont montré que les taux d'aneuploïdie dans le sperme des populations d'hommes infertiles présentant une oligospermie sévère ou modérée étaient plus élevés que ceux des hommes fertiles avec spermogramme normal $[6,18,24]$, et que les taux de disomies des spermatozoïdes étaient environ 20 fois plus élevés dans les ICSI que dans les FIV [45]. Cependant, les résultats de ces différentes études ne permettent pas d'établir un lien évident entre la tératozoospermie polymorphe et la fréquence des disomies et des aneuploïdies dans le sperme.

Les premiers travaux cherchant à montrer une relation potentielle entre la fréquence des anomalies chromosomiques dans le sperme et celle des anomalies morphologiques ont utilisé la technique de fécondation hétérospécifique homme-hamster [32]. Ces travaux n'ont montré aucune corrélation significative entre les anomalies morphologiques et les anomalies chromosomiques des spermatozoïdes. Ceci est vrai à la fois pour la fréquence totale des anomalies, la fréquence des anomalies numériques et celle des anomalies de structure.

L'application de la cytogénétique moléculaire à l'étude du capital génétique des spermatozoïdes permet l'analyse d'un très grand nombre de cellules et l'obtention de résultats fiables. Par cette technique, Rives et al. [41] montrent l'absence significative de corrélation entre la fréquence des dysosmies et la tératozoospermie polymorphe. Dans un élégante étude associant une évaluation du complément chromosomique des spermatozoïdes chez les hommes fertiles par FISH (Fluorescent in situ
Hybridization) à une analyse morphologique en contraste de phase des mêmes spermatozoïdes, Celik-Ozenci et al. [11] démontrent que les anomalies chromosomiques de nombre sont présentes dans les têtes des spermatozoïdes de toutes les tailles et de toutes les formes, des spermatozoïdes diploïdes pouvant même être d'apparence tout à fait normale. De même, des spermatozoïdes à complément chromosomique normal peuvent aussi être de toutes les formes et de toutes les tailles. Enfin, même dans une population à risque chromosomique élevé dans les spermatozoïdes (tels que les porteurs de translocations et les patients traités pour cancer), les anomalies chromosomiques dans le sperme ne s'accompagnent pas d'une augmentation de la fréquence des anomalies morphologiques [11].

Inversement, deux études consacrées à une éventuelle association entre anomalies chromosomiques et tératozoospermie $[30,47]$ montrent une légère augmentation des aneuploïdies dans les tératozoospermies polymorphes, identique à celle observée dans les oligo-asthéno-tératozoospermies, et considérée comme un marqueur de la défaillance de la spermatogenèse. En comparant individuellement les taux de dysosmie dans le sperme de chaque patient infertile avec celui d'une population d'hommes fertiles, Faure et al. [18] montrent aussi que la tératospermie sévère (moins de $5 \%$ de spermatozoïdes normaux) peut accompagner une non disjonction chromosomique méiotique.

L'augmentation significative des aneuploïdies dans le sperme semble plutôt restreinte à une catégorie limitée de tératozoospermie se manifestant au niveau de la tête des spermatozoïdes. Ainsi, dans la globozoospermie, il existe une augmentation significative mais modérée des aneuploïdies des chromosomes acrocentriques et des chromosomes sexuels [36], tandis que l'on rencontre des taux élevés d'anomalies de ségrégation des chromosomes, conduisant à des taux d'aneuploïdies, parfois proches de $100 \%$, dans le syndrome des spermatozoïdes macrocéphales [1] comme cela est rapporté dans les articles consacrés aux tératospermies monomorphes [Mitchell et al., pages 35-45 ; Metzer-Guillemain et al., pages : 46-49]. De même, une légère augmentation des aneuplö̈dies a été observée chez les patients présentant au moins $30 \%$ de noyaux allongés [40].

Globalement, on peut conclure que l'analyse morphologique des spermatozoïdes en microscopie optique, au grossissement habituellement utilisé pour la réalisation des spermocytogrammes (x1000), n'est donc pas un bon indicateur des anomalies chromosomiques dans le sperme humain, excepté dans quelques cas rares dont nous venons de parler.

\section{MORPHOLOGIE DES SPERMATOZOÏDES ET APOPTOSE}

Bien que la relation entre la morphologie des spermatozoïdes et l'apoptose ne soit pas encore parfaitement caractérisée, un nombre croissant de travaux établissent une corrélation positive entre l'infertilité masculine et la présence de marqueurs apoptotiques sur les spermatozoïdes. Tous les paramètres traditionnels du sperme ont été comparés au pourcentage d'apoptose dans le sperme éjaculé, il a ainsi été démontré que le paramètre morphologie était particulièrement lié à l'apoptose. Siddighi et al. [43] puis Chen et al. [13] ont mis en évidence une corrélation négative significative entre la proportion de sper- 
matozoïdes apoptotiques chez des patients respectivement sélectionnés pour infertilité et oligo-asthéno-tératozoospermie, et celle des spermatozoïdes à morphologie normale en utilisant les critères stricts de Tygerberg d'analyse des spermatozoïdes, et comme marqueur de l'apoptose le test de diffusion de l'ADN.

La relation entre marqueurs apoptotiques et morphologie des spermatozoïdes a été démontrée dans une autre étude impliquant cinquante donneurs de sperme [4]. Dans cette étude originale, les spermatozoïdes de chaque donneur ont été séparés en deux fractions, apoptotique et non apoptotique. Pour cela, les spermatozoïdes ont été sélectionnés à l'aide d'un trieur de cellules en fonction de la présence ou de l'absence d'annexine $\mathrm{V}$, la fraction annexine-négative étant non-apoptotique, la fraction annexine-positive étant apoptotique. Les marqueurs apoptotiques étaient le niveau d'activation de la caspase 3 et l'évaluation du potentiel de membrane mitochondrial. Cette étude a montré que le pourcentage moyen des spermatozoïdes dont la morphologie est normale est plus élevé dans les fractions de spermatozoïdes non-apoptotiques que dans les fractions apoptotiques. Les fractions non-apoptotiques ont en particulier un pourcentage moyen de spermatozoïdes avec têtes irrégulières et anomalies de l'acrosome et de la pièce intermédiaire inférieur à ceux des fractions apoptotiques. La relation entre l'apoptose et la morphologie des spermatozoïdes a encore été mise en évidence par d'autres tests d'apoptose tels que le pourcentage de spermatozoïdes avec externalisation de la phosphatidyl sérine, l'expression du récepteur Fas à la surface des spermatozoïdes, l'expression de p53, et le test de fragmentation de la chromatine (TUNEL) [8]. Cette relation a aussi été étudiée et montrée dans d'autres espèces que l'humain.

L'ensemble de ces résultats démontre qu'il existe bien une corrélation positive significative entre la morphologie des spermatozoïdes et l'expression des marqueurs apoptotiques dans le sperme éjaculé. De tous ces travaux, et si l'on tient aussi compte des autres paramètres du sperme corrélés au phénomène d'apoptose, on peut conclure que la sub-population des spermatozoïdes non apoptotiques est de qualité supérieure à celle du groupe des spermatozoïdes apoptotiques.

\section{CONCLUSION}

S'il a été clairement démontré que les taux de fécondation et de clivage, et la qualité embryonnaire étaient significativement diminués lorsque la qualité du sperme décroît, il est assez difficile, excepté lorsque le spermocytogramme met en évidence une anomalie monomorphe des spermatozoïdes, de décider du choix de la technique d'AMP, d'évaluer les risques d'échec de la technique choisie et de définir une conduite à tenir en cas de grossesse, à partir de la seule analyse morphologique des spermatozoïdes faite dans les conditions habituelles d'analyse du sperme.

Dans les cas de tératospermie polymorphe, la persistance avec une fréquence élevée des anomalies de la tête des spermatozoïdes après un test de sélection au percoll oriente le couple vers une ICSI plutôt qu'une FIV classique. Mais la mise en évidence d'une augmentation de la fréquence des anomalies chromosomiques dans le sperme de ces patients à des taux rapportés par Faure et al. [18] ne justifie pas de poser l'indication d'un diagnostic prénatal en cas de grossesse.

II est donc indéniable que l'analyse morphologique des spermatozoïdes est irremplaçable mais que ses résultats ne doi- vent pas être pris en compte isolément, en particulier pour le choix de la technique d' AMP [25].

Par ailleurs cette analyse morphologique nécessite dans des cas précis d'être considérablement affinée par des technologies qui permettent d'accéder à des détails que seuls des grossissements supérieurs à ceux utilisés lors de la réalisation des spermocytogrammes peuvent obtenir. C'est le cas de la microscopie électronique qui permet de visualiser l'organisation de la chromatine et des structures internes du spermatozoïde inaccessibles à la microscopie optique. C'est aussi le cas du MSOME par lequel les spermatozoïdes peuvent être grossis plus de 6000 fois, et qui met en évidence des anomalies qu'on ne peut percevoir avec les grossissements habituellement utilisés, comme les vacuoles nucléaires. Cette technique (IMSI), associée à l'ICSI paraît améliorer de façon significative les taux d'implantation et de grossesse [5]. Ces deux techniques et leurs indications seront détaillées dans un autre article du présent numéro [Albert et al., pages : 26-34].

Nous avons vu, dans le présent article, les réelles difficultés que présente l'analyse morphologique du sperme humain, avec pour conséquences une grande variabilité dans les résultats entre les laboratoires ainsi que dans les études qui tentent de corréler les anomalies spermatiques à d'autres paramètres tels que les taux de fécondation en FIV ou en ICSI, ou les anomalies chromosomiques. II est devient donc indispensable d'envisager une standardisation des procédures employées, de la réalisation des frottis au rendu des résultats, comme le suggèrent Auger et Eustache [2].

\section{REFERENCES}

1. ACHARD V., PAULMYER-MACROIX O., MERCIER G. et al. : Reproductive failure in patients with various percentages of macronuclear spermatozoa : high level of aneuploid and polyploid spermatozoa. J. Androl., 2007, $28: 600-606$.

2. AUGER J., EUSTACHE F. : Standardisation de la classification morphologique des spermatozoïdes humains selon la méthode de David modifiée. Andrologie, 2000, $120: 358-373$.

3. AZIZ N., BUCHAN I., TAYLOR C. et al. : The sperm deformity index: a reliable predictor of the outcome of oocyte fertilization in vitro. Fertil. Steril., 1996, $66: 1000-1008$.

4. AZIZ N., SAID T., PAASCH U. et al. : The relationship between human sperm apoptosis, morphology and the sperm deformity index. Hum. Reprod., 2007, $22: 1413-1419$.

5. BERKOVITZA., ELTES F., YAARI S. et al. : The morphological normalcy of the sperm nucleus and pregnancy rate of intracytoplasmic injection with morphologically selected sperm. Hum. Reprod., 2005, $20: 185-190$.

6. BERNARDINI L., BORINI A., PRETI S. et al. : Study of aneuploidy in normal and abnormal germ cells from semen of fertile and infertile men. Hum. Reprod., 1998, 13 : 3406-3413.

7. BONDE J.P., ERNST E., JENSEN T.K. et al. : Relation between semen quality and fertility : A population-based study of 430 firstpregnancy planners. Lancet, 1998, 352 : 1172-1177.

8. BORINI A., TAROZZI N., BIZZARO D. et al. : Sperm DNA fragmentation : paternal effect on early post-implantation embryo development an ART. Hum. Reprod., 2006, 11 : 2876-2881.

9. BRONSON R., BRONSON S., OULA L. : Ability of abnormally-shaped human spermatozoa to adhere and penetrate zona-free hamster eggs : correlation with sperm morphology and post-incubation motility. J. Androl., 2007, $28:$ 698-705.

10. BUJAN L., MIEUSSET R., MONDINAT C. et al. : Sperm morpho- 
logy in fertile men and its age related variation. Andrologia, 1988, $20: 121-128$.

11. CELIK-OZENCI C., JAKABA., KOVACS T. et al. : Sperm selection for ICSI : shape properties do not predict the absence or presence of numerical chromosomal aberrations. Hum. Reprod., 2004, 19 : 2052-2059.

12. CHECK J.H., BOLLENDORF A., WILSON C. et al. : A retrospective comparison of pregnancy outcome following conventional oocyte insemination vs intracytoplasmic sperm injection for isolated abnormalities in sperm morphology using strict criteria. J. Androl., 2007, $28: 607-612$.

13. CHEN Z., HAUSER R., TRBOVICH A.M. et al. : The relationship between human semen characteristics and sperm apoptosis : a pilot study. J. Androl., 2006, $27:$ 112-120.

14. CHOW V., CHEUNG A.P. : Male infertility. J. Reprod. Med., 2006, $51: 149-156$.

15. COETZEE K., KRUGE T.F., LOMBARD C.J. : Predictive value of normal sperm morphology : a structured literature review. Hum. Reprod. Update, 1998, $4: 73-82$.

16. DAVID G., BISSON J.P., CZYGLIK P. et al. : Anomalies morphologiques du spermatozoïde humain. 1) Propositions pour un système de classification. J. Gyn. Obst. Biol. Reprod., 1975, 4, Suppl 1 : 17-36.

17. ELIASSON R. : Standard for the investigation of human semen. Andrologia, 1971, $3:$ 49-64.

18. FAURE A.K., AKNIN-SEIFER I., FRÉROT G. et al. : Predictive factors for an increased risk of sperm aneuploidies in oligo-asthenoteratozoospermic males. Int. J. Androl., 2007, $30:$ 153-162.

19. FRANKEN D.R., BASTIAAN H.S., KIDSON A. et al. : Zona pellucida mediated acrosome reaction and sperm morphology. Andrologia, 1997, $29: 311-317$.

20. FREDERICSSON B. : Morphologic evaluation of spermatozoa in different laboratories. Andrologia, 1979, $11: 57-61$.

21. FREUND M. : Standards for the rating of human sperm morphology. A cooperative study. Int. J. Fertil., 1966, 11 : 97-110.

22. HAIDL G., SCHILL W.B. : Sperm morphology in fertile men. Arch. Androl., 1993, 31 : 153-157.

23. GUNALP S., ONCULOGLU C., GURGAN T. et al. : A study of semen parameters with emphasis on sperm morphology in a fertile population : an attempt to develop clinical thresholds. Hum. Reprod., 2001, 16 : 110-114.

24. KAHRAMAN S., FINDIKLI N., BIRICIK A. et al. : Preliminary FISH studies on spermatozoa and embryos in patients with variable degrees of teratozoospermia and a history of poor prognosis. Reprod. Biomed. Online, 2006, $12: 752-761$.

25. KEEGAN B.R., BARTON S., SANCHEZ X. et al. : Isolated teratozoospermia does not affect in vitro fertilization outcome and is not an indication for sperm injection. Fertil. Steril., 2007, sous presse.

26. KRUGER T.F., MENKVELD R., STANDER F.S. et al. : Sperm morphologic features as a prognostic factor in in vitro fertilization. Fertil. Steril., 1986, 46 : 1118-1123.

27. KRUGER T.F., ACOSTA A.A., SIMMONS M.S. et al. : Predictive value of abnormal sperm morphology in in vitro fertilization. Fertil. Steril., 1988, 1 :112-117.

28. KRUGER T.F., DUTOIT C.T., FRANKEN D.R. : A new computerized method of reading sperm morphology (strict criteria) is as efficient as technician reading. Urol. Androl., 1993, 59 : 202-209.

29. KUBO-IRIE M., MATSUMIYA K., IWAMOTO T. et al. : Morphological abnormalities in the spermatozoa of fertile and infertile men. Mol. Reprod. Dev., 2005, $70:$ 70-81.

30. MACHEV N., GOSSET P., VIVILLE S. : Chromosome abnormalities in sperm from infertile men with normal somatic karyotypes : teratozoospermia. Cytogenet. Genome Res., 2005, 111 : 352-357.

31. MANSOUR R.T., ABOULGHAR M.A., SEROUR G.I. et al. : The effect of sperm parameters on the outcome of intracytoplasmic sperm injection. Fertil. Steril., 1995, 64 : 982-986.

32. MARTIN R.H., RADEMAKER A. : The relationship between sperm chromosomal abnormalities and sperm morphology in humans. Mutat. Res., 1988, 207 : 159-164.

33. MENKVELD R., STANDER F.S., KOTZE T.J. et al. : The evaluation of morphological characteristics of human spermatozoa according to stricter criteria. Hum. Reprod., 1990, $5: 586-592$.

34. MENKVELD R., KRUGER T.F. : Advantages of strict (Tygerberg) criteria for evaluation of sperm morphology. Int. J. Androl., 1995, 18 Suppl $2: 36-42$.

35. MENKVELD R., WONG W.Y., LOMBARD C.J. et al. : Semen parameters, including WHO and strict criteria morphology, in a fertile and subfertile population : an effort towards standardization of in vivo thresholds. Hum. Reprod., 2001, 16 : 1165-1171.

36. MOREL F., DOUET-GUILBERT N., MEORMAN A. et al. : Chromosome aneuploidy in the spermatozoa of two men with globozoospermia. Mol. Hum. Reprod., 2004, 10 : 835-838.

37. NAGY Z.P., LIU J., JORIS H. et al. : The result of inrtracytoplasmic sperm injection is not related tyo any of the three basic sperm parameters. Hum. Reprod., 1995, $10: 1123-1129$.

38. NALLELLA K.P., SHARMA R.K., AZIZ N. et al. : Significance of sperm characteristics in the evaluation of male infertility. Fertil. Steril., 2006, $85: 629-634$.

39. OMBELET W., BOSMANS E., JANSSEN M. et al. : Semen parameters in a fertile versus subfertile population : A need for change in the interpretation of semen testing. Hum. Reprod., 1997, 12 : 987-993.

40. PRISANT N., ESCALIER D., SOUFIR J.C. et al. : Ultrastructural nuclear defects and increased chromosome aneuploidies in spermatozoa with elongated heads. Hum. Reprod., 2007, 22 : 10521059.

41. RIVES N., SAINT CLAIR A., MAZURIER S. et al. : Relationship between clinical phenotype, semen parameters and aneuploidy frequency in sperm nuclei of 50 infertile males. Hum. Genet., 1999, $105: 266-272$.

42. SCHWARTZ D., MAYAUX M.J., GUIHARD-MOSCATO M.L. et al : Study of sperm morphologic characteristics in a group of 833 fertile men. Andrologia, 1984, $16: 423-428$.

43. SIDDIGHI S., PATTON W.C., JACOBSON J.D. et al : Correlation of sperm parameters with apoptosis assessed by dual fluorescence DNA integrity assay. Arch. Androl., 2004, 50 : 311-314.

44. SODERLUND B., LUNDIN K. : Choosing fertilization method by analysing sperm morphology or by performing swim-up preparation. Acta Obstet. Gynecol. Scand., 2006, 85 : 306-311.

45. STORENG R.T., PLACHOT M., THEOPHILE D. et al. : Incidence of sex chromosome abnormalities in spermatozoa from patients entering an IVF or ICSI protocol. Acta Obstet. Gynecol. Scand., 1998, $77: 191-197$.

46. SUKCHAROEN N., SITHIPRAVEJ T., PROMVIENGHAI S. et al. : Sperm morphology evaluated by computer (IVOS) cannot predict the fertilization rate in vitro after intracytoplasmic sperm injection. Fertil. Steril., 1998, $69:$ 564-568.

47. SUN F., KO E., MARTIN R. : Is there relationship between sperm chromosome abnormalities and sperm morphology. Reprod. Biol. Endocr., 2006, 4 : 1 -5.

48. SVALANDER P., JAKOBSSON A.H., FORSBERG A.S. et al : The outcome of intracytoplasmic sperm injection is unrelated to "strict criteria" sperm morphology. Hum. Reprod., 1996, 11 : 1019-1022.

49. TASDEMIR I., TASDEMIR M., TAVUKCUOGLU S. et al. : Effect of abnormal sperm head morphology on the outcome of intracytoplasmic sperm injection in humans. Hum. Reprod., 1997, 6 : 12141217.

50. TONER J.P., MOSSAD H., GROW D.R. et al. : Value of sperm morphology assessed by strict criteria for prediction of the outcome 
of artificial (intrauterine) insemination. Andrologia, 1995, 27 : 143148.

51. DE VOS A., VAN DE VELDE H., JORIS $H$. et al. : Influence of individual sperm morphology on fertilization, embryo morphology, and pregnancy outcome of intracytoplasmic sperm injection. Fertil. Steril., 2003, $79: 42-48$.

52. WILLIAMS W.W. : Sterility, the Diagnostic Survey of the Infertile Couple. Springfieid, W.W. Williams inc., $1964: 9$.

53. WORLD HEALTH ORGANIZATION : WHO Laboratory Manual for the Examination of Human Semen and Semen-cervical Mucus Interaction, 1st to 4th edn. Cambridge, Cambridge University press, 1980.

54. WORLD HEALTH ORGANIZATION : WHO Laboratory Manual for the Examination of Human Semen and Semen-cervical Mucus Interaction, 1st to 4th edn. Cambridge, Cambridge University press, 1987.

55. WORLD HEALTH ORGANIZATION : WHO Laboratory Manual for the Examination of Human Semen and Semen-cervical Mucus Interaction, 1st to 4th edn. Cambridge, Cambridge University press, 1992.

56. WORLD HEALTH ORGANIZATION : WHO Laboratory Manual for the Examination of Human Semen and Semen-cervical Mucus Interaction, 1st to 4 th edn. Cambridge, Cambridge University press, 1999.

Manuscrit reçu : novembre 2007 ; accepté novembre 2007.

\author{
ABSTRACT \\ What is the value of light microscopy sperm \\ morphological examination?
}

Marie-Roberte GUICHAOUA, Jeanne PERRIN, Cendrine GEOFFROY-SIRAUDIN, Mireille PAPADACCI

The value of sperm morphology to predict the sperm fertilizing capacity is a subject of ongoing debate. However, it is clear that sperm morphological examination is essential to determine sperm quality as part of the assessment of male or couple infertility. Moreover, application of a new high-power magnification method, which allows the choice of spermatozoa with a preferred nuclear morphology, is positively correlated with a dramatic increase in IVF-IMSI pregnancy rates. Several detailed classification systems of sperm abnormalities have been proposed over the last fifty years and each revision of these classifications introduces stricter criteria. Three of these classifications are generally used as reference classifications: the Kruger/Tygerberg classification and the David classification, carefully revised by Auger and Eustache to ensure quality assurance in reproduction biology. However, the results of sperm analyses are very heterogeneous in terms of the overall percentage of morphological abnormalities and the respective frequencies of the various abnormalities. This examination must therefore be performed very carefully based on strictly defined criteria for the assess- ment of each abnormality with harmonization of these criteria between the various observers in the same laboratory and between laboratories.

Various studies have examined the impact of isolated teratozoospermia on the results of IVF and ICSI, but once again with sometimes contradictory results. However, most studies show that the percentage of morphologically normal sperm is positively correlated with the results of ICSI, and many authors agree that a percentage of morphologically normal sperm less than $5 \%$ is predictive of low fertilization and pregnancy rates in IVF and ICSI.

Over the last ten years, it has been shown that aneuploidy rates in the semen of populations of infertile men with moderate or severe oligospermia were higher than those in fertile men with normal sperm counts, and that sperm disomy rates were about 20-fold higher in ICSI than in IVF. However, the results of these various studies fail to demonstrate an obvious link between polymorphic teratozoospermia and the frequency of disomy and aneuploidy in sperm.

Consequently, light microscopy sperm morphological examination, at the magnifications generally used for sperm counts (x 2000), is therefore not a good indicator of chromosomal abnormalities in human semen, except in the rare cases of monomorphic abnormalities. However, this is not the case for the link between sperm morphology and apoptosis, as a growing number of studies establish a positive correlation between male infertility and the presence of apoptotic markers on spermatozoa, and morphological parameters appear to be closely correlated with apoptosis. Finally, standardization of examination procedures and reporting of the results of sperm morphological examination is absolutely essential.

Key words : sperm morphology, infertility, chromosomal abnormalities, apoptosis, IVF, ICSI 